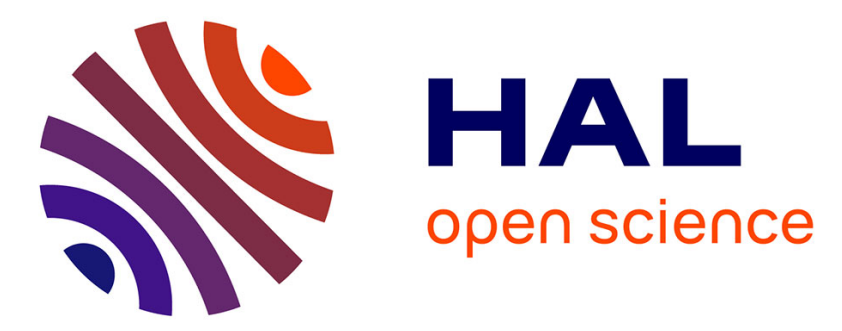

\title{
Balanced Proper Orthogonal Decomposition Applied to Magnetoquasistatic Problems Through a Stabilization Methodology
}

Laurent Montier, Thomas Henneron, Benjamin Goursaud, Stéphane Clenet

\section{- To cite this version:}

Laurent Montier, Thomas Henneron, Benjamin Goursaud, Stéphane Clenet. Balanced Proper Orthogonal Decomposition Applied to Magnetoquasistatic Problems Through a Stabilization Methodology. IEEE Transactions on Magnetics, 2017, 53 (3), pp.1-10. 10.1109/TMAG.2017.2683448 . hal01519726

\author{
HAL Id: hal-01519726 \\ https://hal.science/hal-01519726
}

Submitted on 9 May 2017

HAL is a multi-disciplinary open access archive for the deposit and dissemination of scientific research documents, whether they are published or not. The documents may come from teaching and research institutions in France or abroad, or from public or private research centers.
L'archive ouverte pluridisciplinaire HAL, est destinée au dépôt et à la diffusion de documents scientifiques de niveau recherche, publiés ou non, émanant des établissements d'enseignement et de recherche français ou étrangers, des laboratoires publics ou privés. 


\title{
Balanced Proper Orthogonal Decomposition Applied to Magnetoquasistatic Problems Through a Stabilization Methodology
}

\author{
Laurent Montier ${ }^{1,2}$, Thomas Henneron ${ }^{3}$, Benjamin Goursaud ${ }^{2}$ \\ and Stéphane Clénet ${ }^{1}$ \\ ${ }^{1}$ Univ. Lille, Centrale Lille, Arts et Métiers Paris Tech, HEI, EA 2697 - L2EP \\ Laboratoire d'Electrotechnique et d'Electronique de Puissance, F-59000 Lille, France \\ ${ }^{2}$ EDF R\&D, THEMIS, 7 Boulevard Gaspard Monge, 91120 Palaiseau, France
}

\begin{abstract}
Model Order Reduction (MOR) methods are applied in different areas of physics in order to reduce the computational time of large scale systems. It has been an active field of research for many years, in mechanics especially, but it is quite recent for magnetoquasistatic problems. Although the most famous method, the Proper Orthogonal Decomposition (POD) has been applied for modelling many electromagnetic devices, this method can lack accuracy for low order magnitude output quantities, like flux associated with a probe in regions where the field is low. However, the Balanced Proper Orthogonal Decomposition (BPOD) is a MOR method which takes into account these output quantities in its reduced model to render them accurately. Even if the BPOD may lead to unstable reduced systems, this can be overcome by a stabilization procedure. Therefore, the POD and stabilized BPOD will be compared on a 3D linear magnetoquasistatic field problem.
\end{abstract}

Index Terms-Balanced Proper Orthogonal Decomposition, Balanced Truncation, Model Order Reduction, Proper Orthogonal Decomposition, Stabilization

\section{INTRODUCTION}

C OUPLING the Finite Element Method (FEM) with a time-stepping scheme is more and more used to model electromagnetic devices. Its accuracy has proven to be sufficient over the past few years. However, this method also requires solving large scale systems which have a significant computational cost. To overcome this problem, MOR methods are more and more used since they allow to reduce the size of the system, by projecting it into a so-called reduced basis. Therefore, the quality of the resulting reduced system highly depends on the reduced basis, and thus on the choice of the MOR method.

However, reduced systems sometimes lack robustness: a reduction procedure may lead to an unstable reduced system even if the original system is stable [1] [2]. Moreover, reduced models might produce inaccurate output quantities, which can be very problematic for automatic control systems.

The most used MOR procedure is the Proper Orthogonal Decomposition (POD) [3]. This method can be applied to a multitude of linear and nonlinear problems, and produce in the most cases stable systems. Therefore, the POD is a robust and easy-to-use MOR method. In the field of computational Electromagnetic, the POD has been recently applied to many problems, such as Magnetodynamic problems [4] [5], Electroquasistatic field simulations [6] and Magnetostatic field problems [7]. The POD approach consists in looking for the solution in a reduced basis which concentrates the most energetic states. Therefore, the POD may not be the most suited method for approximating local and low order of magnitude quantities, such as magnetic fluxes associated with probes [8].

To overcome this problem, one may apply the Balanced Proper Orthogonal Decomposition (BPOD) [9]. This method which arises from both the POD method and the Balanced Truncation in Control Theory [10] allows to make a re- duced system which produce high-fidelity output quantities. The BPOD has already been successfully applied to antenna problems [8]. However, the BPOD may unexpectedly also leads to unstable systems [2].

In this article, we propose to develop these two reduction methods, and then to present a methodology which stabilizes and adds robustness to the MOR approaches. Finally, the proposed methods will be compared on a 3D linear academic example.

\section{Magnetoquasistatic Field Problem}

Let us consider a linear magnetoquasistatic field problem defined on a domain $D \subset \mathbb{R}^{3}$ of boundary $\Gamma$ containing a conducting domain $D_{c}$ of boundary $\Gamma_{c} . n_{s}+n_{p}$ stranded coils are included in the domain $D$, where $n_{s}$ denotes the number of source inductors and $n_{p}$, the number of magnetic flux probes. The source inductors are supplied by currents $i_{k}, k=1 \ldots n_{s}$ and the probes are used to measure magnetic fluxes denoted $\Phi_{k}, k=1 \ldots n_{p}$.

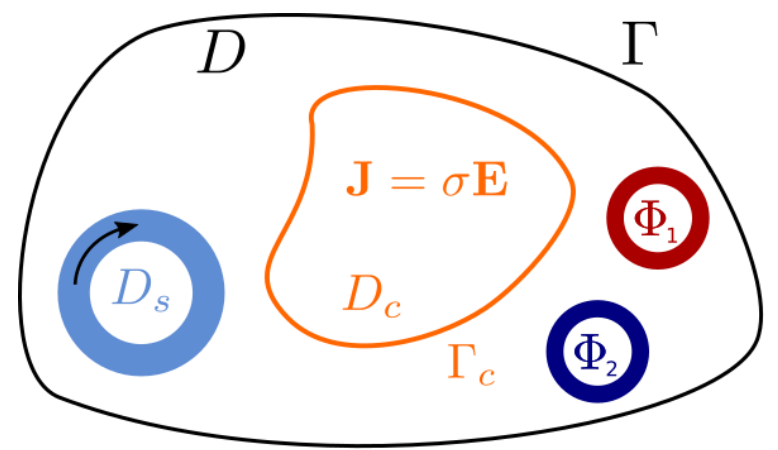

Fig. 1. Domain of the magnetoquasistatic problem 
In the magnetoquasistatic field approximation, without any charge density, displacement currents are not considered. The Maxwell equations describing this problem are then:

$$
\begin{aligned}
\operatorname{curl} \boldsymbol{H}(\boldsymbol{x}, t) & =\sum_{k=1}^{n_{s}} \boldsymbol{N}_{s, k}(\boldsymbol{x}) i_{k}(t)+\boldsymbol{J}_{c}(\boldsymbol{x}, t) \\
\operatorname{curl} \boldsymbol{E}(\boldsymbol{x}, t) & =-\partial_{t} \boldsymbol{B}(\boldsymbol{x}, t) \\
\operatorname{div} \boldsymbol{B}(\boldsymbol{x}, t) & =0
\end{aligned}
$$

where $\boldsymbol{H}$ denotes the magnetic field, $\boldsymbol{B}$ is the magnetic flux density and $\boldsymbol{E}$, the electric field. $\boldsymbol{J}_{c}$ stands for the eddy current density in the conducting domain $D_{c}$ whereas $\boldsymbol{N}_{s, k}, k=$ $1 \ldots n_{s}$, represent the unit current density vector associated with the source inductors.

In order to solve these equations, one has to add some constitutive relations. The first one, named the Ohm law (4), links the vector field describing the eddy current density $\boldsymbol{J}_{c}$ to the electric field $\boldsymbol{E}$ whereas the second one (5) adds a relation between the magnetic field $\boldsymbol{H}$ and the magnetic flux density $\boldsymbol{B}$. The behaviour of the materials are assumed to be linear and isotropic. Therefore, we have:

$$
\begin{aligned}
& \boldsymbol{J}_{c}=\sigma \boldsymbol{E} \\
& \boldsymbol{B}=\mu \boldsymbol{H}
\end{aligned}
$$

where $\mu \in \mathbb{R}^{+*}$ is the magnetic permeability, and $\sigma \in \mathbb{R}^{+}$, the conductivity. In the non-conducting domain $D \backslash D_{c}$, the conductivity is assumed to be null.

To obtain existence and uniqueness of the solution, boundary conditions are to be added. On the boundaries of the domains $D$ and $D_{c}$, they are written:

$$
\begin{array}{ccc}
\boldsymbol{B} \cdot \boldsymbol{n}=0 & \text { on } \quad \Gamma \\
\boldsymbol{J}_{c} \cdot \boldsymbol{n}=0 & \text { on } \quad \Gamma_{c}
\end{array}
$$

Furthermore, the domain $D$ is assumed to be "sufficiently regular" in the sense that the Maxwell-Gauss equation (3) allows to introduce a so-called vector potential such that:

$$
\boldsymbol{B}(\boldsymbol{x}, t)=\operatorname{curl} \boldsymbol{A}(\boldsymbol{x}, t)
$$

In this paper, the modified potential vector formulation is used [11]. Thus, the electric field in $D_{c}$ can be written under the form:

$$
\boldsymbol{E}(\boldsymbol{x}, t)=-\partial_{t} \boldsymbol{A}(\boldsymbol{x}, t)
$$

Finally, a quite strong boundary condition is added:

$$
\boldsymbol{A} \times \boldsymbol{n}=0 \quad \text { on } \Gamma
$$

which guarantee (6).

The solution of the problem is sought on a time domain $[0, T]$. Therefore, the vector potential must belong to $H^{1}\left([0, T] ; X^{0}(D)\right)$ which means that $\boldsymbol{A}$ and its time derivative are $\boldsymbol{L}^{2}([0, T])$ with respect to the time variable, and that $\boldsymbol{A}\left(x, t_{i}\right) \in X^{0}(D) \quad \forall t_{i} \in[0, T]$, where:

$$
\begin{aligned}
& X(D)=\left\{\boldsymbol{A} \in \boldsymbol{L}^{2}(D) ; \operatorname{rot} \boldsymbol{A} \in \boldsymbol{L}^{2}(D) ; \boldsymbol{A} \times \boldsymbol{n}=\left.0\right|_{\Gamma}\right\} \\
& X^{0}(D)=\left\{\boldsymbol{A} \in X(D) ;(\boldsymbol{A}, \nabla \psi)=0, \forall \psi \in \boldsymbol{H}_{0}^{1}(D)\right\}
\end{aligned}
$$

By combining the previous relations, the quasistatic formulation to be solved is then [12]:
Find $\boldsymbol{A} \in H^{1}\left(0, T ; X^{0}(D)\right)$ such that

$$
\begin{array}{r}
\int_{D} \mu^{-1} \operatorname{curl} \boldsymbol{A}(\boldsymbol{x}, t) \cdot \operatorname{curl} \boldsymbol{A}^{\prime}(\boldsymbol{x}) \mathbf{d} \boldsymbol{x}+\int_{D_{c}} \sigma \partial_{t} \boldsymbol{A}(\boldsymbol{x}, t) \cdot \boldsymbol{A}^{\prime}(\boldsymbol{x}) \mathbf{d} \boldsymbol{x} \\
=\int_{D} \sum_{k=1}^{n_{s}} \boldsymbol{N}_{s, k}(\boldsymbol{x}) i_{k}(t) \cdot \boldsymbol{A}^{\prime}(\boldsymbol{x}) \mathbf{d} \boldsymbol{x}
\end{array}
$$

$\forall \boldsymbol{A}^{\prime} \in X(D)$ verifying $\operatorname{div} \boldsymbol{A}^{\prime} \in \mathrm{L}^{2}(D)$

In this problem the boundary condition (6) is essential and (7) is natural [12].

The Finite Element Method is applied to solve this problem by discretizing the vector potential $\boldsymbol{A}$ with the Nedelec edge elements:

$$
\boldsymbol{A}(\boldsymbol{x}, t)=\sum_{i=k}^{N} A_{k}(t) \boldsymbol{w}_{k}^{1}(\boldsymbol{x})
$$

where $N$ denotes the number of edges on the mesh, $\boldsymbol{w}_{k}^{1}(\boldsymbol{x})$ the $k^{t h}$ edge function associated with the $k^{t h}$ edge element. The unknown vector field of components $A_{k}(t), k=1 \ldots N$ is denoted by $\boldsymbol{X}(t)$ and the output vector field $\boldsymbol{Y}(t)$ of size $n_{p}$ is composed of the different fluxes $\Phi_{k}(t), k=1 \ldots n_{p}$ associated to the probe inductors. By introducing $\boldsymbol{N}_{p, k}$, the unit current density vector associated with the $k^{t h}$ probe, the magnetic flux $\phi_{k}$ is expressed as:

$$
\phi_{k}(t)=\int_{D} \boldsymbol{N}_{p, k}(\boldsymbol{x}) \cdot \boldsymbol{A}(\boldsymbol{x}, t) \mathbf{d} \boldsymbol{x}
$$

Therefore, the problem (11-13) can be written through a system of differential algebraic equations (DAE):

$$
\begin{aligned}
& \boldsymbol{K} \frac{\mathrm{d} \boldsymbol{X}(t)}{\mathrm{d} t}+\boldsymbol{M} \boldsymbol{X}(t)=\boldsymbol{F}_{s r c} \boldsymbol{I}(t) \\
& \boldsymbol{Y}(t)=\boldsymbol{F}_{p r b}^{t} \boldsymbol{X}(t)
\end{aligned}
$$

with $\boldsymbol{I}(t) \in \mathbb{R}^{n_{s}}$ the input vector field of which its components are the currents applied to the source inductors. $\boldsymbol{M}$ and $\boldsymbol{K}$ are positive semidefinite matrice sof size $N$. $\boldsymbol{F}_{s r c} \in \mathbb{R}^{N \times n_{s}}$ is a matrix in which are concatenated the unitary current density vectors of the sources inductors. $\boldsymbol{F}_{p r b} \in \mathbb{R}^{N \times n_{p}}$ denotes its counterpart, accounting for the probe inductors. Their expressions are:

$$
\begin{aligned}
K_{i j} & =\int_{D_{c}} \sigma \boldsymbol{w}_{i}^{1}(\boldsymbol{x}) \cdot \boldsymbol{w}_{j}^{1}(x) \mathbf{d} \boldsymbol{x} \\
M_{i j} & =\int_{D} \mu^{-1} \boldsymbol{r o t} \boldsymbol{w}_{i}(\boldsymbol{x}) \cdot \boldsymbol{r o t} \boldsymbol{w}_{j}^{1}(x) \mathbf{d} \boldsymbol{x} \\
\left(F_{s r c}\right)_{i j} & =\int_{D} \boldsymbol{N}_{s, j}(\boldsymbol{x}) \cdot \boldsymbol{w}_{i}^{1}(\boldsymbol{x}) \mathbf{d} \boldsymbol{x} \\
\left(F_{p r b}\right)_{i j} & =\int_{D} \boldsymbol{N}_{p, j}(\boldsymbol{x}) \cdot \boldsymbol{w}_{i}^{1}(\boldsymbol{x}) \mathbf{d} \boldsymbol{x}
\end{aligned}
$$

In order to get the solution of the discrete problem (14-15), a time-stepping scheme such as the Backward Euler Method may be used. By using the notation $\boldsymbol{u}\left(t_{k}\right)=\boldsymbol{u}^{k}$ with $t_{k}$ being a time step given by $t_{k}=k \tau$, problem (14-15) reads:

$$
\begin{aligned}
& \left(\frac{\boldsymbol{K}}{\tau}+\boldsymbol{M}\right) \boldsymbol{X}^{k+1}=\boldsymbol{F}_{s r c} \boldsymbol{I}^{k}+\frac{\boldsymbol{K}}{\tau} \boldsymbol{X}^{k} \\
& \boldsymbol{Y}^{k+1}=\boldsymbol{F}_{p r b}^{t} \boldsymbol{X}^{k+1}
\end{aligned}
$$


On the other hand, if the transient response is not desired, the harmonic problem can be formulated as followed:

$$
\begin{aligned}
& (j \omega \boldsymbol{K}+\boldsymbol{M}) \boldsymbol{X}(\omega)=\boldsymbol{F}_{s r c} \boldsymbol{I}(\omega) \\
& \boldsymbol{Y}(\omega)=\boldsymbol{F}_{p r b}^{t} \boldsymbol{X}(\omega)
\end{aligned}
$$

where $\omega$ is the angular frequency.

\section{Model ORder Reduction With BPOD AND POD}

In this section, the Balanced Truncation, on which the BPOD is based, is firstly developed. Then, the BPOD and the POD approaches are presented in order to derive from the full model a reduced model which is accurate for any input signal of frequency within the bandwidth $\Omega=\left[\omega_{A}, \omega_{B}\right]$, through a greedy algorithm.

Furthermore, these three methods were proposed for systems where $\boldsymbol{K}$ and $\boldsymbol{M}$ are symmetric definite matrices, which is not the case with our problem. However, we will present this method by assuming this point, and we will propose a generalization in the section for semi-definite matrices.

\section{A. Balanced Truncation Method}

The BPOD has arisen from the Balanced Truncation method introduced by Moore [13]. This method is based on Control Theory in order to find a reduced basis with an accurate output response. Given a generalized multi-input/multi-output (MIMO) state-space system such as (14-15), with a statevector $\boldsymbol{X}(t) \in \mathbb{R}^{N}$, an input vector $\boldsymbol{I}(t) \in \mathbb{R}^{n_{s}}$ and an output vector $\boldsymbol{Y}(t) \in \mathbb{R}^{n_{p}}$, the first step is to define Controllability and Observability Gramians. Those quantities, denoted as $\mathcal{G}_{c}$ and $\mathcal{G}_{o}$ are positive definite matrices of size $N \times N$. Their expression in the frequency domain are:

$$
\begin{aligned}
& \mathcal{G}_{\boldsymbol{c}}=\int_{0}^{\infty}(j \omega \boldsymbol{K}+\boldsymbol{M})^{-1} \boldsymbol{F}_{s r c} \boldsymbol{F}_{s r c}^{*}\left(-j \omega \boldsymbol{K}^{*}+\boldsymbol{M}^{*}\right)^{-1} \mathrm{~d} \omega \\
& \mathcal{G}_{\boldsymbol{o}}=\int_{0}^{\infty}\left(j \omega \boldsymbol{K}^{*}+\boldsymbol{M}^{*}\right)^{-1} \boldsymbol{F}_{p r b} \boldsymbol{F}_{p r b}^{*}(-j \omega \boldsymbol{K}+\boldsymbol{M})^{-1} \mathrm{~d} \omega
\end{aligned}
$$

where $\boldsymbol{X}^{*}$ denotes the complex conjugate transpose of $\boldsymbol{X}$. As stated at the beginning of this section, the square matrices involved ( $\boldsymbol{K}$ and $\boldsymbol{M}$ ) are assumed to be positive definite (the semi-definite case will be treated in the following). On FEM systems arising from magnetoquasistatic problems such as (14-15), the right-hand-side matrices are real. Therefore, these definitions reduce to:

$$
\begin{aligned}
& \mathcal{G}_{\boldsymbol{c}}=\int_{0}^{\infty}(j \omega \boldsymbol{K}+\boldsymbol{M})^{-1} \boldsymbol{F}_{s r c} \boldsymbol{F}_{s r c}^{t}(-j \omega \boldsymbol{K}+\boldsymbol{M})^{-1} \mathrm{~d} \omega \\
& \mathcal{G}_{\boldsymbol{o}}=\int_{0}^{\infty}(j \omega \boldsymbol{K}+\boldsymbol{M})^{-1} \boldsymbol{F}_{p r b} \boldsymbol{F}_{p r b}^{t}(-j \omega \boldsymbol{K}+\boldsymbol{M})^{-1} \mathrm{~d} \omega
\end{aligned}
$$

Gramians are coordinate dependent, i.e. they depend on the basis in which they are expressed. Thus, balancing a system consists in finding a basis $\mathcal{R}$, in which both the Gramians are diagonal and equal. By denoting with "tilde" quantities expressed in the balanced basis, the following equality holds:

$$
\begin{gathered}
\widetilde{\mathcal{G}_{c}}=\mathcal{R}^{-1} \mathcal{G}_{c}\left(\mathcal{R}^{-1}\right)^{*}, \quad \widetilde{\mathcal{G}_{o}}=\mathcal{R}^{*} \mathcal{G}_{o} \mathcal{R} \\
\widetilde{\mathcal{G}_{c}}=\widetilde{\mathcal{G}_{o}}=\Lambda
\end{gathered}
$$

where $\boldsymbol{\Lambda}$ is a real diagonal positive matrix of size $N$ which contains the so-called Hankel Singular Values.

Finally, the Balanced Truncation approach consists in selecting the most significant vectors of this basis, i.e. which produce the highest Hankel Singular Values. Thus, the least significant vectors are truncated from the balanced basis. The remaining vectors are called the balanced reduced basis.

However, finding a balanced reduced basis requires an eigenvalue decomposition of the Gramians product $\mathcal{G}_{c} \mathcal{G}_{o}$ [14]. This computation becomes unaffordable for large scale systems because of two main reasons:

- computing and constructing both Gramians defined by (26-27) is too expensive in terms of computation time and memory requirements

- performing an eigenvalue decomposition is very demanding for large scale systems

To overcome these difficulties, the formalism of Proper Orthogonal Decomposition computes directly an approximation of this balanced reduced basis while keeping a decent computational cost. This method is the Balanced Proper Orthogonal Decomposition.

\section{B. Balanced Proper Orthogonal Decomposition}

The Balanced Proper Orthogonal Decomposition approach introduced by Willcox [9] is based on a low-rank approximation of both Gramians, through the method of snapshots.

1) Controllability snapshots

Let $\boldsymbol{X}_{s r c, \boldsymbol{k}}^{\omega_{i}}$ be the solution of the harmonic system (22-23) at the angular frequency $\omega_{i}$ when only the $k^{t h}$ source inductor is supplied by a current of $1 A$. This vector is called a snapshot because it represents a solution of the system for particular values of the input parameters, i.e. the angular frequency and the $n_{s}$ currents in the inductors. It follows that this snapshot is also the $k^{t h}$ column of the matrix $(j \omega \boldsymbol{K}+\boldsymbol{M})^{-1} \boldsymbol{F}_{s r c} \in$ $\mathbb{C}^{N \times n_{s}}$. Let $\boldsymbol{X}_{s r c}^{\omega_{i}}$ be the matrix in which are concatenated $\boldsymbol{X}_{s r c, \boldsymbol{k}}^{\omega_{i}}$ :

$$
\begin{aligned}
\boldsymbol{X}_{s r c}^{\omega_{i}} & =\left(\boldsymbol{X}_{s r c, \mathbf{1}}^{\omega_{i}}, \boldsymbol{X}_{s r c, \mathbf{2}}^{\omega_{i}}, \ldots, \boldsymbol{X}_{s r c, \boldsymbol{n}_{\boldsymbol{s}}}^{\omega_{i}}\right) \\
& =(j \omega \boldsymbol{K}+\boldsymbol{M})^{-1} \boldsymbol{F}_{s r c}
\end{aligned}
$$

Using (26) and (29), the Controllability Gramian reads:

$$
\mathcal{G}_{c}=\int_{0}^{\infty} \boldsymbol{X}_{s r c}^{\omega}\left(\boldsymbol{X}_{s r c}^{\omega}\right)^{*} \mathrm{~d} \omega
$$

Then, the integral over all frequencies in (31) is approximated using a sum over $m$ training angular frequencies $\omega_{i}, i=$ $1 \ldots m$. Therefore:

$$
\mathcal{G}_{\boldsymbol{c}} \approx \sum_{k=1}^{m} \boldsymbol{X}_{s r c}^{\omega_{k}}\left(\boldsymbol{X}_{s r c}^{\omega_{k}}\right)^{*} \delta_{k}
$$


where $\delta_{k}$ are quadrature weights. In practice however, it is possible to set $\delta_{k}=1$ [10]. Thus, after applying the complexconjugate relation, the Controllability Gramian is:

$$
\mathcal{G}_{\boldsymbol{c}} \approx \sum_{k=1}^{m}\left[\operatorname{Re}\left(\boldsymbol{X}_{s r c}^{\omega_{k}}\right) \operatorname{Re}\left(\boldsymbol{X}_{s r c}^{\omega_{k}}\right)^{t}+\operatorname{Im}\left(\boldsymbol{X}_{s r c}^{\omega_{k}}\right) \operatorname{Im}\left(\boldsymbol{X}_{s r c}^{\omega_{k}}\right)^{t}\right]
$$

which by introducing the real matrix $\boldsymbol{X}_{s r c} \in \mathbb{R}^{N \times 2 m n_{s}}$ defined as the concatenation of the real and imaginary parts of the different snapshots, is rewritten as:

$$
\mathcal{G}_{c} \approx \boldsymbol{X}_{s r c} \boldsymbol{X}_{s r c}^{t}
$$

with

$$
\boldsymbol{X}_{s r c}=\left(\left.\operatorname{Re}\left(\boldsymbol{X}_{s r c}^{\omega_{i}}\right)\right|_{i=1 \ldots m},\left.\operatorname{Im}\left(\boldsymbol{X}_{s r c}^{\omega_{i}}\right)\right|_{i=1 \ldots m}\right)
$$

2) Observability snapshots

The same procedure is used on the dual system of (14-15) in order to approximate the Observability Gramian $\mathcal{G}_{\boldsymbol{o}}$. With $\boldsymbol{K}$ and $\boldsymbol{M}$ being real symmetric on one hand, $\boldsymbol{F}_{s r c}$ and $\boldsymbol{F}_{p r b}$ being real on the other hand, the dual system reduces to:

$$
\begin{aligned}
& \boldsymbol{K} \frac{\mathrm{d} \widehat{\boldsymbol{X}(t)}}{\mathrm{d} t}+\boldsymbol{M} \widehat{\boldsymbol{X}}(t)=\boldsymbol{F}_{p r b} \widehat{\boldsymbol{I}}(t) \\
& \widehat{\boldsymbol{Y}}(t)=\boldsymbol{F}_{s r c}^{t} \widehat{\boldsymbol{X}}(t)
\end{aligned}
$$

with the "hat" notation expressing dual quantities. Basically, probes are now sources of the system, and vice versa. To summarize, the two steps to perform are:

1) Compute $\boldsymbol{X}_{p r b, \boldsymbol{k}}^{\omega_{i}}$ the harmonic solution of the dual system (34-35) at the $m$ training angular frequencies $\omega_{i}$ when only the $k^{\text {th }}$ probe inductor is supplied by a current of $1 A$. Then, define $\boldsymbol{X}_{\text {prb }}^{\omega_{i}} \in \mathbb{C}^{N \times n_{p}}$, the counterpart of $\boldsymbol{X}_{\text {src }}^{\omega_{i}} \in \mathbb{C}^{N \times n_{s}}$.

$$
\begin{aligned}
\boldsymbol{X}_{p r b}^{\omega_{i}} & =\left(\boldsymbol{X}_{p r b, \mathbf{1}}^{\omega_{i}}, \boldsymbol{X}_{p r b, \mathbf{2}}^{\omega_{i}}, \ldots, \boldsymbol{X}_{p r b, \boldsymbol{n}_{p}}^{\omega_{i}}\right) \\
& =\left(j \omega_{i} \boldsymbol{K}+\boldsymbol{M}\right)^{-1} \boldsymbol{F}_{p r b}
\end{aligned}
$$

2) Define $\boldsymbol{X}_{p r b} \in \mathbb{R}^{N \times 2 m n_{p}}$ as:

$$
\boldsymbol{X}_{p r b}=\left(\left.\operatorname{Re}\left(\boldsymbol{X}_{p r b}^{\omega_{i}}\right)\right|_{i=1 \ldots m},\left.\operatorname{Im}\left(\boldsymbol{X}_{p r b}^{\omega_{i}}\right)\right|_{i=1 \ldots m}\right)
$$

As for the Controllability Gramian, the Observability Gramian is then easily expressed by:

$$
\mathcal{G}_{o} \approx \boldsymbol{X}_{p r b} \boldsymbol{X}_{p r b}^{t}
$$

\section{3) Balanced reduced basis}

Once both Gramians are expressed as a Cholesky form as in (32) and (38), computing the balanced reduced basis is achieved efficiently through the Singular Value Decomposition (SVD) on the real rectangular matrix $\boldsymbol{M}_{\text {correl }}=\boldsymbol{X}_{p r b}^{t} \boldsymbol{X}_{s r c} \in$ $\mathbb{R}^{2 m n_{p} \times 2 m n_{s}}$. Let $r$ be the rank of $\boldsymbol{M}_{\text {correl }}$. Then, the SVD of $\boldsymbol{M}_{\text {correl }}$ is:

$$
\begin{aligned}
\boldsymbol{M}_{\text {correl }} & =\left(\begin{array}{ll}
\boldsymbol{U}_{1} & \boldsymbol{U}_{2}
\end{array}\right)\left(\begin{array}{cc}
\boldsymbol{\Sigma} & 0 \\
0 & 0
\end{array}\right)\left(\begin{array}{l}
\boldsymbol{V}_{1}^{t} \\
\boldsymbol{V}_{2}^{t}
\end{array}\right) \\
& =\boldsymbol{U}_{1} \boldsymbol{\Sigma} \boldsymbol{V}_{1}^{t}
\end{aligned}
$$

where $\boldsymbol{\Sigma} \in \mathbb{R}^{r \times r}$ a real positive diagonal matrix, $\boldsymbol{U}_{1} \in$ $\mathbb{R}^{2 m n_{p} \times r}$ and $\boldsymbol{V}_{1} \in \mathbb{R}^{2 m n_{s} \times r}$ which verify:

$$
\boldsymbol{U}_{1}^{t} \boldsymbol{U}_{1}=\boldsymbol{I}_{r}, \quad \boldsymbol{V}_{1}^{t} \boldsymbol{V}_{1}=\boldsymbol{I}_{r}
$$

with $\boldsymbol{I}_{r}$ the identity matrix of size $r$.

Finally, the truncated balanced reduced basis is obtained with:

$$
\begin{aligned}
& \boldsymbol{T}=\boldsymbol{X}_{s r c} \boldsymbol{V}_{1} \boldsymbol{\Sigma}^{-1 / 2} \\
& \boldsymbol{S}=\boldsymbol{\Sigma}^{-1 / 2} \boldsymbol{U}_{1}^{t} \boldsymbol{X}_{p r b}^{t}
\end{aligned}
$$

$\boldsymbol{T} \in \mathbb{R}^{N \times r}$ defines the $r$ first vectors of the full basis $\mathcal{R}$ from the balanced truncation (28), whereas $S \in \mathbb{R}^{r \times N}$ is its counterpart for the inverse transformation $\mathcal{R}^{-1}$ [14]. Moreover, this basis ensures that the Gramians $\widetilde{\mathcal{G}_{c}}$ and $\widetilde{\mathcal{G}_{o}}$ are equal and diagonal, which is the definition of a balanced reduced basis. Indeed, by substituting $\boldsymbol{T}$ for $\mathcal{R}$ and $S$ for $\mathcal{R}^{-1}$, we have according to (28), (32) and (42):

$$
\widetilde{\mathcal{G}_{c}}=S_{\mathcal{G}_{c}} \boldsymbol{S}^{t}=\boldsymbol{\Sigma}
$$

and by using (28), (38) and (41), one can find:

$$
\widetilde{\mathcal{G}_{o}}=\boldsymbol{T}^{t} \mathcal{G}_{o} \boldsymbol{T}=\boldsymbol{I}_{r}
$$

With this reduced basis, both Gramians are of size $r$, the dimension of the reduced basis, and no longer of size $N$, with $r<<N$.

\section{4) Reduced system}

In the model order reduction framework, the solution is sought into a subspace defined by a reduced basis of size $r$, usually very small in front of the dimension of the full system $N$. Therefore, the key tool of the BPOD approach is to use both the balanced reduced basis in order to obtain an accurate reduced system.

First, we look for a reduced basis $\Psi \in \mathbb{R}^{N \times r}$ such that we have an accurate approximation:

$$
\boldsymbol{X}(t) \approx \boldsymbol{\Psi} \boldsymbol{X}_{r}(t)
$$

where $\boldsymbol{X}_{r}(t)$ is the reduced vector field solution, of size $r, r<<N$. Then, the balanced reduced basis related to the Controllability Gramian $\boldsymbol{T}$ appears as a good candidate since it contains snapshots of the primal system. Thus, we write:

$$
\boldsymbol{X}(t) \approx \boldsymbol{T} \boldsymbol{X}_{r}(t)
$$

Therefore, injecting the latter equation (44) into the full problem $(14-15)$ leads to:

$$
\begin{aligned}
& \boldsymbol{K} \boldsymbol{T} \frac{\mathrm{d} \boldsymbol{X}_{r}(t)}{\mathrm{d} t}+\boldsymbol{M} \boldsymbol{T} \boldsymbol{X}_{r}(t)=\boldsymbol{F}_{s r c} \boldsymbol{I}(t) \\
& \boldsymbol{Y}(t)=\boldsymbol{F}_{p r b}^{t} \boldsymbol{T} \boldsymbol{X}_{r}(t)
\end{aligned}
$$

System (45-46) has $N$ equations with only $r$ unknowns. In order to solve it, one can cancel the projection of the residue onto a reduced basis. Then, the BPOD approach consists in taking the balanced reduced basis related to the Observability Grammian $S$ in order to obtain accurate output quantities. Therefore, multyplying (45-46) by $S$ gives a system with $r$ equations and $r$ unknowns:

$$
\begin{aligned}
& \boldsymbol{S} \boldsymbol{K} \boldsymbol{T} \frac{\mathrm{d} \boldsymbol{X}_{r}(t)}{\mathrm{d} t}+\boldsymbol{S} \boldsymbol{M} \boldsymbol{T} \boldsymbol{X}_{r}(t)=\boldsymbol{S F}_{s r c} \boldsymbol{I}(t) \\
& \boldsymbol{Y}(t)=\boldsymbol{F}_{p r b}^{t} \boldsymbol{T} \boldsymbol{X}_{r}(t)
\end{aligned}
$$


which can be rewritten as:

$$
\begin{aligned}
& \boldsymbol{K}_{r} \frac{\mathrm{d} \boldsymbol{X}_{r}(t)}{\mathrm{d} t}+\boldsymbol{M}_{r} \boldsymbol{X}_{r}(t)=\boldsymbol{F}_{s r c, r} \boldsymbol{I}(t) \\
& \boldsymbol{Y}(t)=\boldsymbol{F}_{p r b, r}^{t} \boldsymbol{X}_{r}(t)
\end{aligned}
$$

with $\boldsymbol{K}_{r}=\boldsymbol{S} \boldsymbol{K} \boldsymbol{T} \in \mathbb{R}^{r \times r}, \boldsymbol{M}_{r}=\boldsymbol{S} \boldsymbol{M} \boldsymbol{T} \in \mathbb{R}^{r \times r}, \boldsymbol{F}_{s r c, r}=$ $\boldsymbol{S F}_{s r c}$ and $\boldsymbol{F}_{p r b, r}=\boldsymbol{T}^{t} \boldsymbol{F}_{p r b}$. Equations (49-50) define the reduced system of size $r$.

Remark 1: This kind of projection where the left and the right reduced basis $\boldsymbol{S}$ and $\boldsymbol{T}$ are different is called a PetrovGalerkin projection.

Remark 2: Equations (49-50) can also be optained by applying the change of coordinates $\mathcal{R}$ to the system (14-15) defined in (28) under the assumption that $\mathcal{R} \approx \boldsymbol{T}$ and that $\mathcal{R}^{-1} \approx S$.

\section{Proper Orthogonal Decomposition}

The Proper Orthogonal Decomposition is one of the most famous model order reduction method and was introduced by Lumley in 1967 for fluid mechanics applications [15]. The main idea of the POD is to use snapshots of only the primal system in order to build a reduced system. Therefore, POD naturally derives from the BPOD framework introduced above, with the sole difference that it does not take into account probes nor the dual system (34-35).

Exaclty as for the first step of BPOD, the controllability snapshots $\boldsymbol{X}_{s r c}$ have to be computed as in (33). However, the POD approach does not require doing so with the dual system (34-35): instead of applying a SVD on the correlation matrix $\boldsymbol{M}_{\text {correl }}=\boldsymbol{X}_{o b s}^{t} \boldsymbol{X}_{s r c}$ as in the BPOD, the POD method only considers the auto-correlation matrix $\boldsymbol{M}_{A C}=\boldsymbol{X}_{s r c}^{t} \boldsymbol{X}_{s r c}$. Since $\boldsymbol{M}_{A C}$ is symmetric, the SVD reduces to a classical Eigenvalue Decomposition (EVD).

Therefore, the remaining steps are:

1) Compute the EVD of $\boldsymbol{M}_{A C}=\boldsymbol{U}_{1} \boldsymbol{\Sigma} \boldsymbol{V}_{1}^{t}$

2) Compute the reduced basis:

$$
\begin{aligned}
& \boldsymbol{T}=\boldsymbol{X}_{s r c} \boldsymbol{V}_{1} \boldsymbol{\Sigma}^{-1 / 2} \\
& \boldsymbol{S}=\boldsymbol{\Sigma}^{-1 / 2} \boldsymbol{U}_{1}^{t} \boldsymbol{X}_{\text {scr }}^{t}=\boldsymbol{T}^{t}
\end{aligned}
$$

The left reduced basis reduces to the transpose of the right reduced basis.

3) Form the reduced system (49-50) with the matrix being this time symmetric: with $\boldsymbol{K}_{r}=\boldsymbol{T}^{t} \boldsymbol{K} \boldsymbol{T} \in \mathbb{R}^{r \times r}, \boldsymbol{M}_{r}=$ $\boldsymbol{T}^{t} \boldsymbol{M} \boldsymbol{T} \in \mathbb{R}^{r \times r}, \boldsymbol{F}_{s r c, r}=\boldsymbol{T}^{t} \boldsymbol{F}_{s r c}$ and $\boldsymbol{F}_{p r b, r}=\boldsymbol{T}^{t} \boldsymbol{F}_{p r b}$

Remark 3: POD may be seen as a BPOD variant which ensures that input quantities will be approximated very accurately. In fact, the POD methods reduces to the BPOD method when applied to the following system:

$$
\begin{aligned}
& \boldsymbol{K} \frac{\mathrm{d} \boldsymbol{X}(t)}{\mathrm{d} t}+\boldsymbol{M} \boldsymbol{X}(t)=\boldsymbol{F}_{s r c} \boldsymbol{I}(t) \\
& \boldsymbol{Y}(t)=\boldsymbol{F}_{s r c}^{t} \boldsymbol{X}(t)
\end{aligned}
$$

where one can see that the matrix $\boldsymbol{F}_{s r c}^{t}$ defining the output quantities $\boldsymbol{Y}$ is the transpose of the matrix on which is applied the command in (14).

\section{Greedy algorithm}

Although the construction of the reduced system through POD or BPOD has been detailed, the model order reduction process is not fully developed. Indeed, the quality of the reduced system for a frequency bandwidth $\Omega=\left[\omega_{A}, \omega_{B}\right]$ will depend a lot on the choice of the snapshots, i.e., the training angular frequencies $\omega_{i}, i=1 \ldots m$ chosen to build the reduced basis.

One way to select them is to discretize $\Omega$ into an equispaced set of $p$ angular frequencies $\Omega_{p}=$ $\left\{\omega_{A}+\frac{k}{p-1}\left(\omega_{B}-\omega_{A}\right), k=0 \ldots p-1\right\}$ and then to compute snapshots for every angular frequency $\omega_{i} \in \Omega_{p}$. However, snapshots computation is the most demanding part of the POD or BPOD methods in terms of computation time. Thus, this direct approach may not be the most "optimal" choice because the number of snapshots increases linearly with $p$.

In this paper, we propose to use a greedy algorithm based on the residual vector [16] in order to chose $m<<p$ locally optimal training frequencies amongst the set $\Omega_{p}$. The algorithm will iteratively select these frequencies through the reduced system. Therefore, snapshots computation now scales with $m$ and no longer with $p$. It means that one can use a very fine grid $\Omega_{p}$ while keeping a decent computational cost since only $m<<p$ snapshots computation are needed.

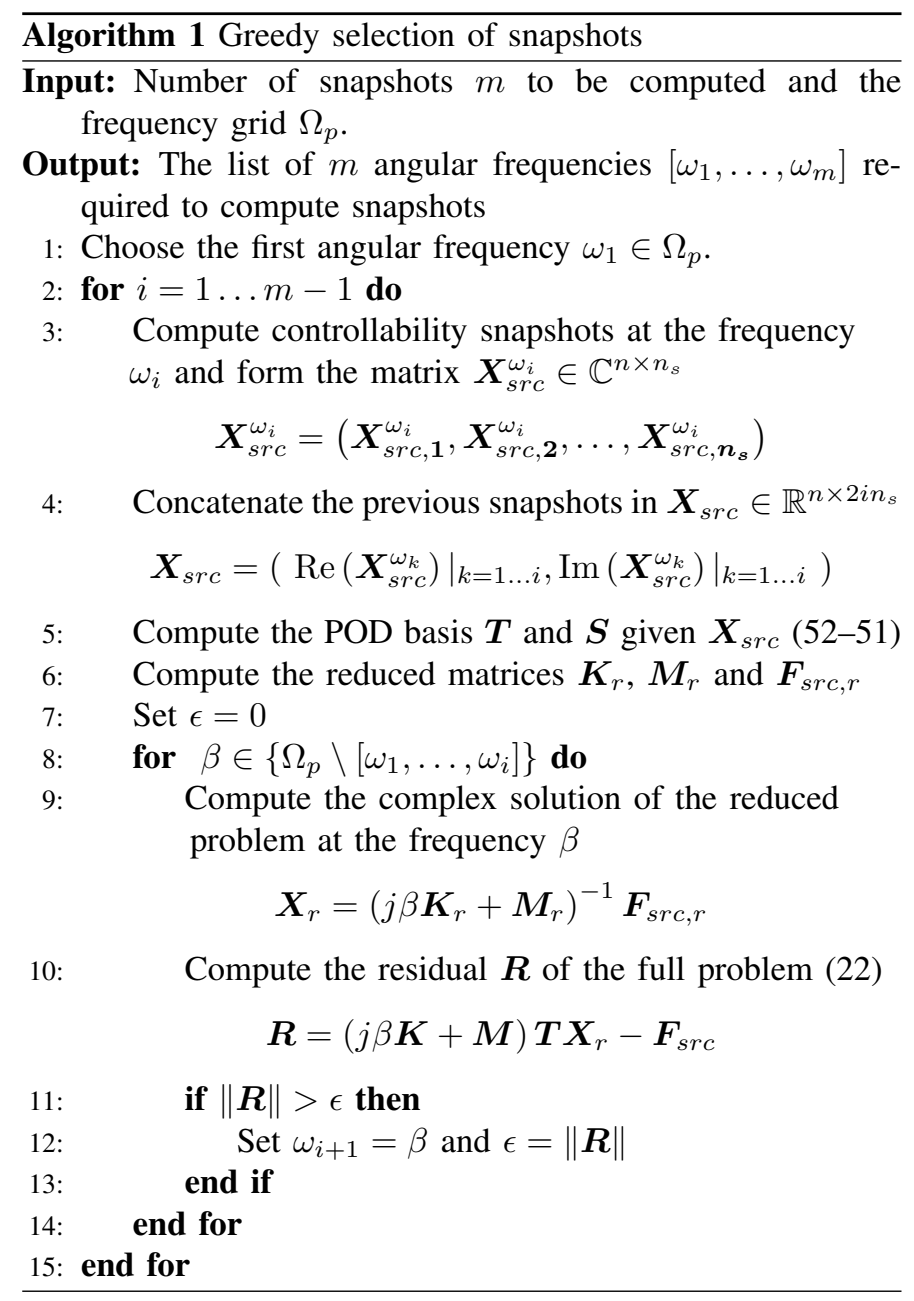




\section{RoBUSTNESS}

In the previous sections, we have shown how to build POD and BPOD reduced system through an efficient choice of training frequencies. However, the reduction procedure may lead to instabilities or numerical errors, and thus, may lack robustness.

The SVD applied to numerical problems, like the ones arising from the FEM, may produce numerical errors: modes associated to the smallest singular values may not be orthogonal and thus, may be related to numerical noise. Truncating according to the order of magnitude of the singular values is often performed in order to reduce this error. But this approach may lack robustness since the truncation depends on an arbitrary truncation coefficient fixed by the user. Moreover, many magnetoquasistatic problems lead to systems where $\boldsymbol{K}$ in (14) is singular, and thus, where we are not in the condition of application of the POD and the BPOD. Therefore, applying model order reduction methods to these systems may lead to instabilities [17]. Finally, performing an oblique projection where the left and right reduced basis $S$ and $T$ are different, such as in the BPOD, can produce instabilities. For instance, a stable system reduced with an oblique projection may lead to an unstable system as defined in subsection IV.B [2].

Hence, methods for improving the robustness regarding to the truncation and the stability are presented in this section.

\section{A. Truncation}

Truncation plays an essential part in approaches like POD or BPOD which are based on the SVD. Actually, the Singular Value Decomposition allows to extract the rank of the matrices $M_{\text {correl }}=\boldsymbol{X}_{p r b}^{t} \boldsymbol{X}_{s r c}$ and $M_{A C}=\boldsymbol{X}_{s r c}^{t} \boldsymbol{X}_{s r c}$ through the size of the matrix $\boldsymbol{\Sigma}$ containing the singular values.

However, for numerical problems such as the ones arising from the FEM, these correlation matrices are likely to be full rank even though their last singular values may be very small. Actually, they might arise from numerical noise. To address this issue, the most common approach is to truncate by replacing singular values in $\boldsymbol{\Sigma}$ with zero if they are smaller to a certain threshold. However, setting this threshold too high will lead to a lack of accuracy and setting it to low may give rise to instabilities. Even though this approach has proven to work pretty well, the choice of the truncation coefficient is very problem dependent. Thus, the user often has to tune it in order to obtain a reduced system which is both accurate and stable.

Hence, the question to address is on how to differentiate a very small singular value which contributes to the dynamic of the system and the spurious zero, which can lead to computational errors. In this paper, we took a different approach. Thus, once the reduction procedure has been applied, (41-42) leads to the following orthogonality condition for a reduced system of size $r$ :

$$
S T=I_{r}
$$

However, this is not always true. In fact, this matrix product is more likely to be equal to:

$$
\boldsymbol{S T}=\left(\begin{array}{cc}
\boldsymbol{I}_{t} & 0 \\
0 & \boldsymbol{\Theta}
\end{array}\right)
$$

where $\Theta$ is not diagonal. Therefore, we propose to use $t$, the size of $\boldsymbol{I}_{t}$ as the size of the truncated reduced system. The new reduced basis $\boldsymbol{T}_{t}$ and $\boldsymbol{S}_{t}$ consist in the first $t$ columns/rows of $\boldsymbol{T}$ and $\boldsymbol{S}$ respectively. In order to keep in the following a methodology for both truncated and untruncated systems, we set $r=t$, the new size of the reduced system.

Finally, the computation time of the matrix-matrix product in (56) is acceptable since $\boldsymbol{S}$ and $\boldsymbol{T}$ are rectangular matrices. Actually, the complexity of this computation is $O\left(N r^{2}\right)$.

Remark 4 In practice, $I_{t}$ in (56) cannot be the perfect identity matrix. However, looking at the matrix product $\boldsymbol{S T}$ allows to see two distinct areas: a first one which is very close to the identity matrix, and a second with non-significant extra-diagonal terms. In order to find $t$, first is to define for $k=1 \ldots r$ the square matrices $\boldsymbol{J}_{k} \in \mathbb{R}^{k \times k}$, composed of the $k$ first rows and columns of $\boldsymbol{S T}$. Then, $t \leq r$ is the biggest integer which verifies $\left\|\boldsymbol{J}_{t}-\boldsymbol{I}_{t}\right\|_{F}<\epsilon_{F}$ where $\|\cdot\|_{F}$ denotes the Frobenius norm, and $\epsilon_{F}$ a user prescribed accuracy.

\section{B. Stability}

In this subsection, stability of reduced systems will be investigated under the assumption that both $\boldsymbol{K}$ and $\boldsymbol{M}$ are positive definite matrices.

\section{1) Definition}

Given a space-time system as (14-15), a system is said to be asymptotically stable if impulse responses become null beyond a certain time:

$$
\begin{array}{cl}
\lim _{t \rightarrow \infty} \boldsymbol{X}(t)=0, & \forall \boldsymbol{X}(t) / \boldsymbol{K} \frac{\mathrm{d} \boldsymbol{X}}{\mathrm{d} t}(t)+\boldsymbol{M} \boldsymbol{X}(t)=0 \\
& \boldsymbol{X}(t=0) \neq 0
\end{array}
$$

The generalized eigenvalues of the pencil $(\boldsymbol{K}, \boldsymbol{M})$ denoted by $\Lambda(\boldsymbol{K}, \boldsymbol{M})$ is an interesting tool for proving stability.

Theorem 1: System (14-15) is asymptotically stable if and only if

$$
\min \{\operatorname{Re}(\lambda), \lambda \in \Lambda(\boldsymbol{K}, \boldsymbol{M})\}>0
$$

where

$$
\lambda \in \Lambda(\boldsymbol{K}, \boldsymbol{M}) \Leftrightarrow \exists \boldsymbol{X} \in \mathbb{R}^{n} / \boldsymbol{K} \boldsymbol{X}=\lambda \boldsymbol{M} \boldsymbol{X}
$$

Therefore, one can investigate the stability of a reduced system only by looking to its generalized eigenvalues.

\section{2) Guaranteed stability with POD}

By assuming that $\boldsymbol{T} \in \mathbb{R}^{n \times r}$ does not contain the null column-vector, one can show that $\boldsymbol{K}_{r}=\boldsymbol{T}^{t} \boldsymbol{K} \boldsymbol{T}$ and $\boldsymbol{M}_{r}=$ $\boldsymbol{T}^{t} \boldsymbol{M T}$ are positive definite as well. Then, the following theorem deriving from the generalized Courant-Fischer theorem [18] ensures the stability of the POD reduced system.

Theorem 2: Let $\boldsymbol{A}$ and $\boldsymbol{B}$ be two positive definite matrices in $\mathbb{R}^{n \times n}$. Then:

$$
\min \{\operatorname{Re}(\lambda), \lambda \in \Lambda(\boldsymbol{A}, \boldsymbol{B})\}>0
$$




\section{3) Stability algorithm with BPOD}

Due to the Petrov-Galerkin projection, stability of the reduced equation (49) is not ensured with the BPOD approach. Indeed, $\boldsymbol{K}_{r}=\boldsymbol{S} \boldsymbol{K} \boldsymbol{T}$ is not even symmetric. Therefore, $\boldsymbol{K}_{r}$ and in the same way, $\boldsymbol{M}_{r}$, are not positive definite and thus, Theorem 2 does not hold.

To overcome this problem, a stabilization algorithm is needed. An elegant stabilization procedure based on SQP optimization is proposed by D. Amsallem and C. Fahrat [2]. However, this algorithm requires a reduced system with $\boldsymbol{K}_{r}=$ $\boldsymbol{I}_{r}$ in (49). In this article, we propose a quite straightforward algorithm based on [2] which will provide a stable reduced system, in the sense that its generalized eigenvalues are positive as defined in the subsection IV.B.1:

Given an unstable reduced system of size $r$, the underlying idea of this algorithm is to find a stable reduced system of size $q, q<r$ within the unstable one. This can be done by constructing $\widetilde{\boldsymbol{T}}$ composed of $q$ column-vectors of the reduced basis $\boldsymbol{T}$ and $\widetilde{\boldsymbol{S}}$, a matrix containing $q$ row-vectors of the reduced basis $\boldsymbol{S}$, such that the reduced system induced by this basis is stable. In practice, one has to construct squared submatrices $\widetilde{\boldsymbol{M}}_{r}$ and $\widetilde{\boldsymbol{K}}_{r}$ in $\mathbb{R}^{q \times q}$ by extracting the same $q$ rows and columns, of index $\gamma_{1} \ldots \gamma_{q}$, of $\boldsymbol{M}$ and $\boldsymbol{K}$ respectively. $\widetilde{\boldsymbol{M}}_{r}$ and $\widetilde{\boldsymbol{K}}_{r}$ must verify the asymptotic stability condition (58). The detailed steps are presented in Algorithm 2.

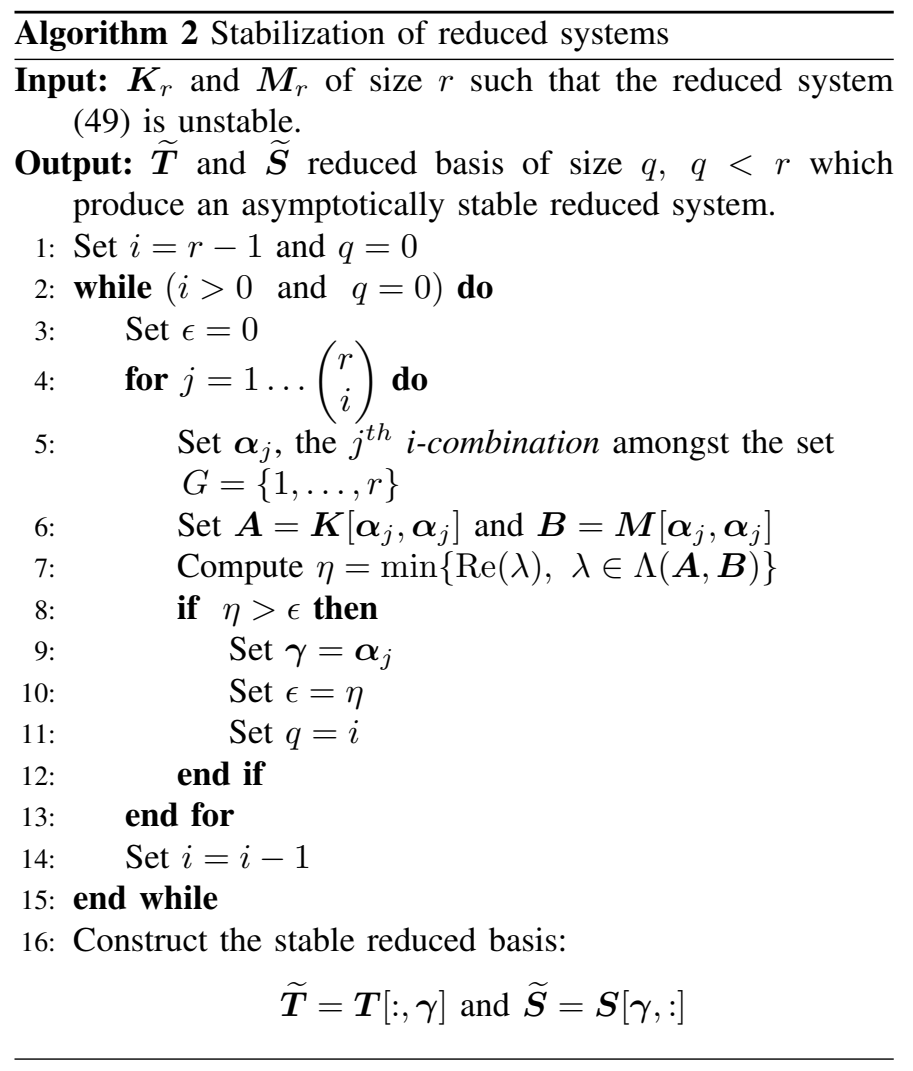

Finally, constructing a reduced system through $\widetilde{\boldsymbol{T}}$ and $\widetilde{\boldsymbol{S}}$ will lead to a stable reduced basis. Moreover, even if the algorithm requires solving generalized eigenvalue problems on $(\boldsymbol{A}, \boldsymbol{B})$ many times, this is not computationally expensive since $\boldsymbol{A}$ and $\boldsymbol{B}$ are matrices of small size.

\section{Differential algebraic equations}

So far, $\boldsymbol{K}$ has been assumed to be a positive definite matrix. However, if the conducting domain $D_{c}$ does not cover the whole domain $D$, as it is generally the case for magnetoquasistatic problems, then $\boldsymbol{K}$ defined by (16) is null except in $D_{c}$. Therefore, $\boldsymbol{K}$ is only positive semidefinite. This is important in terms of properties of the system: if $\boldsymbol{K}$ was definite positive, then the Differential Algebraic Equations (DAE) (14-15) could be transform into an Ordinary Differential Equation (ODE) by applying $\boldsymbol{K}^{-1}$ on (14). Then, the system of DAE (14$15)$ would be equivalent to an ODE in a certain sense. Since $\boldsymbol{K}^{-1}$ is not defined in our case, (14-15) cannot be reduced to an ODE.

When reducing DAE systems, one cannot ensure that the reducing matrix $\boldsymbol{K}_{r}$ is full rank, because one vector of the reduced basis $\boldsymbol{T}$ may lay in the kernel of $\boldsymbol{K}$.

To prevent this issue with the POD procedure, one possibility is to modify the autocorrelation matrix $\boldsymbol{M}_{A C}$. Thus, considering $\boldsymbol{M}_{A C}=\boldsymbol{X}_{s r c}^{t} \boldsymbol{K} \boldsymbol{X}_{s r c}$ instead of $\boldsymbol{M}_{A C}=\boldsymbol{X}_{s r c}^{t} \boldsymbol{X}_{s r c}$ in the POD approach ensures that $\boldsymbol{K}_{r}$ is full rank and equals to $\boldsymbol{I}_{r}$ after truncation.

As for the BPOD, the same methodology works. Considering $\boldsymbol{M}_{\text {correl }}=\boldsymbol{X}_{\text {obs }}^{t} \boldsymbol{K} \boldsymbol{X}_{\text {src }}$ instead of $\boldsymbol{M}_{\text {correl }}=\boldsymbol{X}_{\text {obs }}^{t} \boldsymbol{X}_{\text {src }}$ leads to $\boldsymbol{K}_{r}=\boldsymbol{I}_{r}$.

For sake of clarity, we will further call this approach as the DAE-SVD.

Remark 5: Doing so may reduce the rank of both the correlation and autocorrelation matrices.

Remark 6: This approach ensures that the POD reduced system is asymptotically stable since $\boldsymbol{M}_{r}$ is positive definite, $\boldsymbol{K}_{r}=\boldsymbol{I}_{r}$ as Theorem $\mathbf{2}$ stated below shows.

Remark 7: Using these correlation matrices actually consists in considering only the proper Gramians, quantities defined for DAE systems [19].

Remark 8: If the system is not gauged, then both $M$ and $\boldsymbol{K}$ are not positive definite. However, $\boldsymbol{M}_{r}$ and $\boldsymbol{K}_{r}$ are likely to be positive definite for the POD, and can be stabilized through the use of Algorithm 2.

\section{APPLICATIONS}

\section{A. Magnetoquasistatic problem}

A 3D linear magnetodynamic problem composed of an aluminium conducting plate and three inductors is studied. The first one is the source inductor and is supplied with a square wave signal of frequency $f_{0}=1 \mathrm{kHz}$, whereas the two other plays the role of magnetic flux probes as shown in figure 2. Therefore, system (14-15) has one input signal, and two output signals. The problem is discretized in 3D with 159882 tetrahedrons and has 189412 edge unknowns. The problem is solved with a 3D-FEM code (so called the full order model in the following) in the time domain on six periods $T=1 \mathrm{~ms}$, with a $25 \mu \mathrm{s}$ time step, in order to compare the results to the ones arising from the different reduced systems. The preconditioned conjugate gradient method is used in order to solve the full order model. 


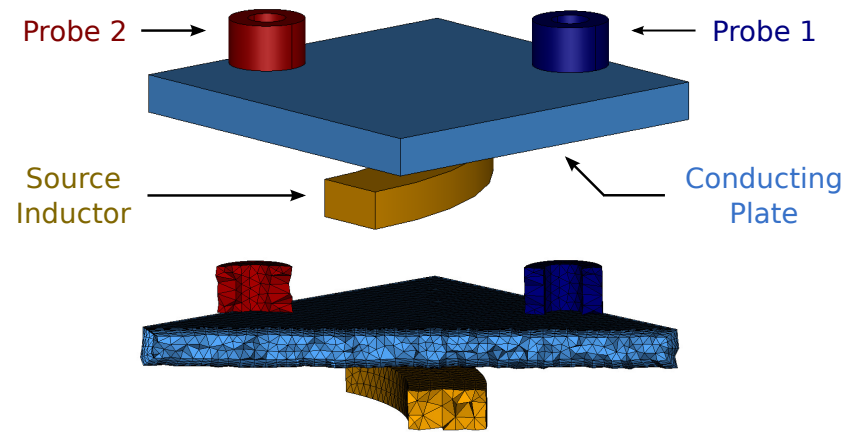

Fig. 2. 3D mesh of the problem

\section{B. Model Order Reduction}

Four reduced models will be compared with a full FEM code on this example. The first two reduced systems consists in the POD and the BPOD as presented in section III. The last two reduced models consists in applying the BPOD, when either the first or the second probe inductor is considered in their model. Those will be referred as SISO 1, and SISO 2, SISO standing for Single Input Single Output. Finally, snapshots are taken in the frequency domain, in the bandwidth $\Omega=[100 \mathrm{~Hz}, 7000 \mathrm{~Hz}]$. The reduced linear system, which is of small size (less than 20 equations) is solved using a direct solver.

Then, the four methods are compared by varying the number of snapshots used to compute the reduced basis. The training angular frequencies $w_{i}, i=1 \ldots m$ have been selected by applying the greedy algorithm (Algorithm 1) on the frequency grid of size $70: \Omega_{70}=\{0.1,0.2, \ldots, 7\} \mathrm{kHz}$. The frequencies $f_{i}=w_{i} / 2 \pi, i=1 \ldots m$ selected are presented in table I.

TABLE I

ANGULAR FREQUENCIES (KHZ) SELECTED THROUGH THE GREEDY ALGORITHM

\begin{tabular}{|c|c|c|c|c|c|c|c|c|c|}
\hline$f_{1}$ & $f_{2}$ & $f_{3}$ & $f_{4}$ & $f_{5}$ & $f_{6}$ & $f_{7}$ & $f_{8}$ & $f_{9}$ & $f_{10}$ \\
\hline 1 & 7 & 0.1 & 3.2 & 0.3 & 5.3 & 0.5 & 1.9 & 0.2 & 6.3 \\
\hline
\end{tabular}

In order to estimate the accuracy of our models, relative errors will be computed on the fluxes associated with the source inductor $\phi_{s r c}$, and the two probe inductors $\phi_{p r b, 1}$ and $\phi_{p r b, 2}$. These relative errors are computed through the error operator $\mathcal{E}(\mathcal{P})$ defined as:

$$
\mathcal{E}(\mathcal{P})=\sqrt{\frac{\int_{0}^{6 T}\left(\mathcal{P}_{\text {red }}(t)-\mathcal{P}_{\text {ref }}(t)\right)^{2} \mathrm{~d} t}{\int_{0}^{6 T} \mathcal{P}_{\text {ref }}(t)^{2} \mathrm{~d} t}}
$$

where $\mathcal{P}_{\text {ref }}$ denotes a scalar quantity computed by the reference code and $\mathcal{P}_{\text {red }}$ stands for a quantity calculated through the reduced system. rithm

1) Model Order Reduction without the stabilization algo-

First, table II shows the behaviour, i.e. stable or unstable, of the reduced systems versus the number of snapshots $m$, when the stabilization procedure defined in Algorithm 2 has not been applied. In this table, "s" stands for stable, and "u" for unstable.
TABLE II

STABILITY ANALYSIS OF REDUCED SYSTEMS

\begin{tabular}{|c|c|c|c|c|c|c|c|c|c|c|}
\hline$m$ & 1 & 2 & 3 & 4 & 5 & 6 & 7 & 8 & 9 & 10 \\
\hline POD & $\mathrm{s}$ & $\mathrm{s}$ & $\mathrm{s}$ & $\mathrm{s}$ & $\mathrm{s}$ & $\mathrm{s}$ & $\mathrm{s}$ & $\mathrm{s}$ & $\mathrm{s}$ & $\mathrm{s}$ \\
\hline BPOD & $\mathrm{s}$ & $\mathbf{u}$ & $\mathbf{u}$ & $\mathrm{s}$ & $\mathrm{s}$ & $\mathrm{s}$ & $\mathrm{s}$ & $\mathbf{u}$ & $\mathrm{s}$ & $\mathbf{u}$ \\
\hline SISO 1 & $\mathrm{~s}$ & $\mathrm{~s}$ & $\mathrm{~s}$ & $\mathrm{u}$ & $\mathrm{s}$ & $\mathrm{s}$ & $\mathrm{s}$ & $\mathrm{s}$ & $\mathrm{s}$ & $\mathrm{s}$ \\
\hline SISO 2 & $\mathrm{~s}$ & $\mathbf{u}$ & $\mathrm{s}$ & $\mathrm{s}$ & $\mathrm{s}$ & $\mathrm{s}$ & $\mathrm{s}$ & $\mathrm{s}$ & $\mathbf{u}$ & $\mathrm{s}$ \\
\hline
\end{tabular}

From table II, one can see that without stabilization, 4 over 10 systems generated by the BPOD method are unstable, while the guaranteed stability of the POD presented in section IV.B.2 is showed.

Therefore, the stabilization algorithm is required for the BPOD or the SISO methods in order to obtain robust reduced models. Thus, the next results will be in the case where Algorithm 2 has been applied.

2) Reduction with the stabilization algorithm

Reduction errors on $\phi_{p r b, 2}$ when the stabilization algorithm has been applied are presented in figure 3. Even though the BPOD procedure shows strange behaviours around 6 snapshots, one can see that the stabilization algorithm works and produce acceptable results, even for the BPOD.

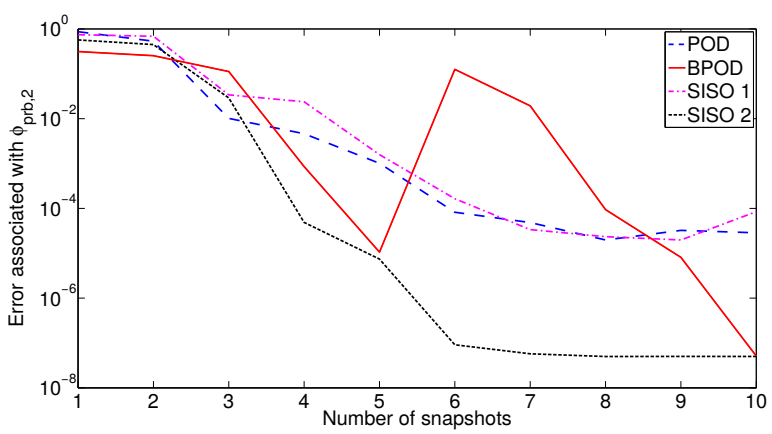

Fig. 3. Reduction error associated with $\phi_{p r b, 2}$, with the regular SVD

3) Reduction error with the DAE-SVD and the stabilization algorithm

Figure 4 shows the reduction error associated with $\phi_{p r b, 2}$ when both the stabilization algorithm and the DAE-SVD have been applied. In this case, the BPOD shows a much better behaviour since the relative error always decreases when the number of snapshots is increasing.

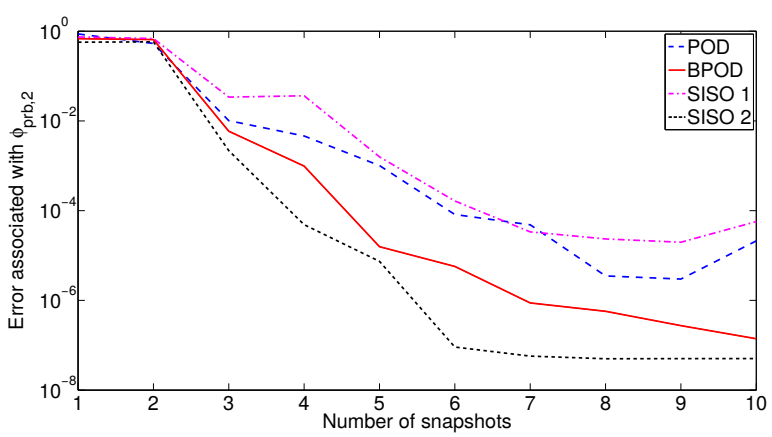

Fig. 4. Reduction error associated with $\phi_{p r b, 2}$, without truncation 


\section{4) Reduction error with the three stabilization procedures}

Finally, computations are done with the three stabilization procedures, i.e. the stabilization algorithm, the DAE-SVD and the truncation. First, figure 5 shows the relative reduction error on the fluxes associated with $\phi_{p r b, 2}$.

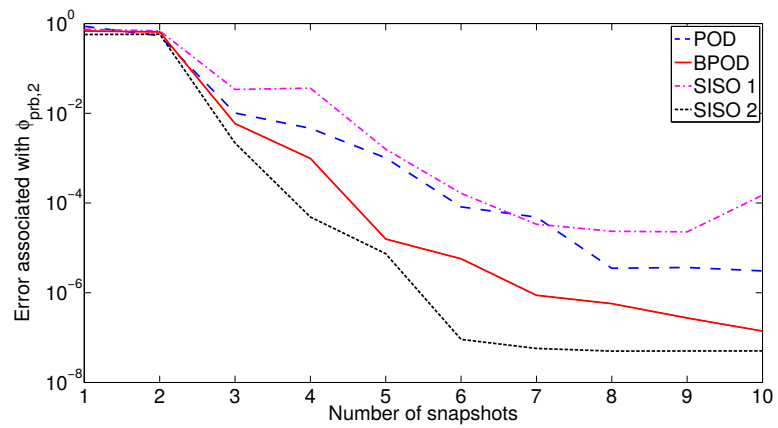

Fig. 5. Reduction error associated with $\phi_{p r b, 2}$

When using the truncation, the POD procedure give slightly better results, whereas the SISO 1 method actually give worse results. Since the snapshots matrices $S$ and $\boldsymbol{T}$ are of small size ( 20 rows/columns at most), the number of modes being truncated for stability considerations is pretty small ( 1 or 2 in practice). Basically, results don't differ much from the ones where the truncation has not been applied because of two reasons:

1) The truncation is often used in model order reduction in order to stabilize systems which can become unstable because of numerical noise. However, since the stabilization algorithm has also been applied, this point may appear irrelevant.

2) The truncation procedure allows to find the rank of the correlation matrix when the snapshots are "almost" not linearly independent. However, since the greedy algorithm has been applied, it is very likely that the correlation matrices are full rank for a low number of snapshots.

Furthermore, figures, 6 and 7 show the relative reduction error on the fluxes associated with the source inductor $\phi_{s r c}$, and the first probe $\phi_{p r b, 1}$ versus the number of snapshots required to compute reduced basis.

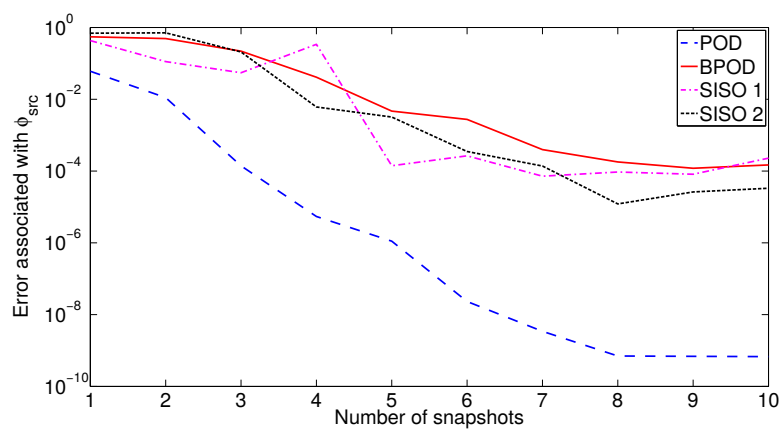

Fig. 6. Reduction error associated with $\phi_{s r c}$

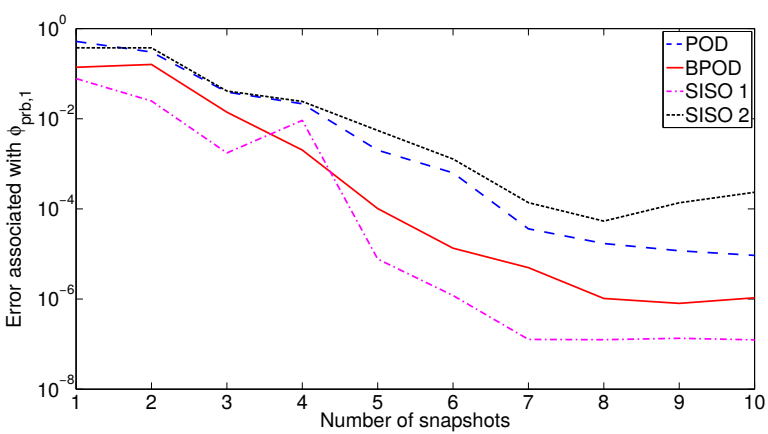

Fig. 7. Reduction error associated with $\phi_{p r b, 1}$

From figure 6, one can see that the POD is much more accurate than the three other methods based on BPOD for approximating the flux associated to the source inductor. Figure 7 shows that the SISO 1 and the BPOD approaches are the most efficient methods for the flux associated with the first probe. As for the flux associated with the second inductor, the SISO 2 and the BPOD approaches are this time the best, as shown in figure 5.

Those results are consistent since the SISO 1 and SISO 2 arise from only taking into account the first and the second inductor respectively in a BPOD approach. Therefore, they produce very precise fluxes associated with the first and the second probe respectively. Moreover, the BPOD gives a good approximation of both probe fluxes. Once more, this is consistent because this method takes into account both probes in its model. Furthermore, it actually makes sense that the POD gives very good results for the flux associated with the source inductor: as explained in Remark 3, the POD approach can be seen as a BPOD variant in which the observed quantity is this source inductor flux.

\section{5) Impact of the Greedy Algorithm}

All the previous computations have been made using the greedy algorithm in order to select the training frequencies. In this subsection, the impact of this algorithm is studied by comparing the results obtained with it when the 3 stabilization procedures are used, with a uniformly distributed training frequencies selection. Since the signal is a square wave of frequency $f_{0}=1 \mathrm{kHz}$, we have decided to use the different harmonics of $f_{0}$, i.e. $f=\left\{f_{0}, 2 f_{0}, \ldots, 10 f_{0}\right\}$.

Figure 8 presents the error associated with the second probe when the greedy algorithm is used or not for both the POD and the BPOD methods (the two SISO methods have been omitted in this case for a sake of clarity).

From figure 8, one can see that the greedy algorithm allows to obtain a better accuracy up to $10-4$ for $\mathrm{m}=10$ snapshots. Maybe the a priori choice of the frequencies to calculate the snapshots was not optimal, but the greedy algorithm is very interesting because the user does not have to define which frequencies to use. All he has to do is to define a frequency range for which the reduced order model has to be valid.

6) Speedups

Speedups are presented in table III. They are computed by taking into account both the construction of the reduced 


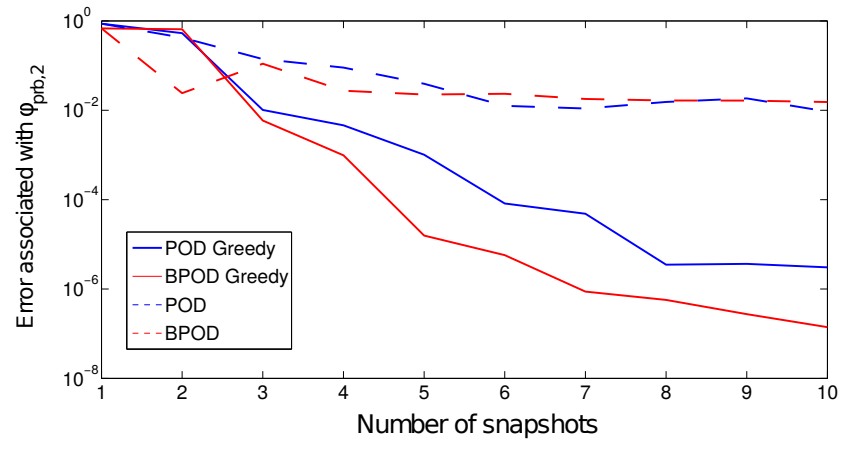

Fig. 8. Reduction error associated with $\phi_{p r b, 2}$

systems and the solution of the reduced problem.

TABLE III

SPEEDUP OF THE REDUCED SYSTEMS

\begin{tabular}{|c|c|c|c|c|c|c|c|c|c|c|}
\hline$m$ & 1 & 2 & 3 & 4 & 5 & 6 & 7 & 8 & 9 & 10 \\
\hline POD & 53 & 33.9 & 13.3 & 11.4 & 8.6 & 7.9 & 6.7 & 6 & 5 & 4.8 \\
\hline BPOD & 20 & 12.7 & 5 & 4.3 & 3.3 & 3 & 2.5 & 2.2 & 1.9 & 1.8 \\
\hline SISO 1 & 27.4 & 17.7 & 7.1 & 6.1 & 4.6 & 4.2 & 3.5 & 3.2 & 2.7 & 2.5 \\
\hline SISO 2 & 23.9 & 19.2 & 7.5 & 6.4 & 4.8 & 4.4 & 3.7 & 3.4 & 2.8 & 2.7 \\
\hline
\end{tabular}

From table III, we can see that POD is faster than the other methods since it does not require solving dual system (34-35).

Furthermore, this table is relevant in terms of comparison between the different methods. Indeed, the speedup is very dependent on the length of the transient state and on the size of the full system. Finally, the BPOD is slower than the POD with a factor of about 2.5 for $m=1 \ldots 10$. This shows that the computational complexity of the BPOD scales with the POD one.

Finally, by looking at the accuracy of the proposed methods versus their speed-up, the POD is the most accurate reduced method: a speed-up of 5 is obtained with $m=9$ training angular frequencies whereas this corresponds to only $m=3$ for the BPOD. Then, the POD is more accurate with $m=9$ than the BPOD with $m=3$. However, when only considering the computational cost associated with the solution of the reduced system, the BPOD is more efficient than the POD. Indeed, the speed-up is in this case related to $m$ since a direct solver is used. The different figures showed in this section reflect this point of view: the accuracy is plotted versus the number of snapshots, i.e. $m$. Then, the BPOD is actually more accurate than the POD from this point of view. This point is important when the reduced system is solved several times (the reduced model is then considered as a metamodel). For example, studying the influence of the waveshape of the excitation coil supply versus the response of the sensor would require many evaluations of the metamodel. In this case, the best achievable accuracy for a given speed-up accounting only for the solution of the reduced system is obtained with the BPOD.

\section{CONCLUSION}

The Proper Orthogonal Decomposition and the Balanced Proper Orthogonal Decomposition applied to linear magne- toquasistatics problems through the modified vector potential formulation have been developed. Moreover, a methodology has been presented in order to produce stable and accurate reduced systems, by trying to use the least arbitrary coefficient: either by using another truncation approach, or by using a greedy algorithm. The 3D example have shown that the POD is robust and very fast, but also that the BPOD through a stabilization procedure produce very accurate results while keeping a decent speedup.

\section{REFERENCES}

[1] Bond, Bradley N and Daniel, Luca, "Guaranteed stable projection-based model reduction for indefinite and unstable linear systems," in Proceedings of the 2008 IEEE/ACM International Conference on ComputerAided Design. IEEE Press, 2008, pp. 728-735.

[2] Amsallem, David and Farhat, Charbel, "Stabilization of projection-based reduced-order models," International Journal for Numerical Methods in Engineering, vol. 91, no. 4, pp. 358-377, 2012.

[3] A. C. Antoulas, D. C. Sorensen, and S. Gugercin, "A survey of model reduction methods for large-scale systems," Contemporary mathematics, vol. 280, pp. 193-220, 2001.

[4] Sato, Yuki and Igarashi, Hajime, "Model reduction of three-dimensional eddy current problems based on the method of snapshots," Magnetics, IEEE Transactions on, vol. 49, no. 5, pp. 1697-1700, 2013.

[5] Pierquin, A. and Henneron, T. and Clénet, S. and Brisset, S., "ModelOrder Reduction of Magnetoquasi-Static Problems Based on POD and Arnoldi-Based Krylov Methods," Magnetics, IEEE Transactions on, vol. 51, no. 3, pp. 1-4, March 2015.

[6] D. Schmidthausler and M. Clemens, "Low-order electroquasistatic field simulations based on proper orthogonal decomposition," IEEE Transactions on Magnetics, vol. 48, no. 2, pp. 567-570, 2012.

[7] Henneron, Thomas and Clenet, Stephane, "Model Order Reduction of Non-Linear Magnetostatic Problems Based on POD and DEI Methods," Magnetics, IEEE Transactions on, vol. 50, no. 2, pp. 33-36, 2014.

[8] Wang, Wei and Paraschos, Georgios N and Vouvakis, Marinos N, "Fast Frequency Sweep of FEM Models via the Balanced Truncation Proper Orthogonal Decomposition," Antennas and Propagation, IEEE Transactions on, vol. 59, no. 11, pp. 4142-4154, 2011.

[9] Willcox, Karen and Peraire, Jaime, "Balanced model reduction via the proper orthogonal decomposition," AIAA journal, vol. 40, no. 11, pp. 2323-2330, 2002.

[10] Phillips, J.R. and Silveira, L.M., "Poor man's TBR: a simple model reduction scheme," Computer-Aided Design of Integrated Circuits and Systems, IEEE Transactions on, vol. 24, no. 1, pp. 43-55, Jan 2005.

[11] Emson, CRI and Simkin, J, "An optimal method for 3-D eddy currents," Magnetics, IEEE Transactions on, vol. 19, no. 6, pp. 2450-2452, 1983.

[12] Nicaise, Serge, "Existence results for the $A-\varphi$ magnetodynamic formulation of the Maxwell system," Tech. Rep., Dec. 2013. [Online]. Available: $\{$ http://hal.archives-ouvertes.fr/hal-00917597\}

[13] Moore, Bruce, "Principal component analysis in linear systems: Controllability, observability, and model reduction," Automatic Control, IEEE Transactions on, vol. 26, no. 1, pp. 17-32, 1981.

[14] Rowley, CW, "Model reduction for fluids, using balanced proper orthogonal decomposition," International Journal of Bifurcation and Chaos, vol. 15, no. 03, pp. 997-1013, 2005.

[15] J. L. Lumley, "The structure of inhomogeneous turbulent flows," Atmospheric turbulence and radio wave propagation, vol. 790, pp. 166-178, 1967.

[16] Hess, Martin W and Benner, Peter, "Fast Evaluation of Time-Harmonic Maxwell's Equations Using the Reduced Basis Method," Microwave Theory and Techniques, IEEE Transactions on, vol. 61, no. 6, pp. 22652274, 2013.

[17] Bai, Zhaojun and Dewilde, Patrick M and Freund, Roland W, "Reducedorder modeling," Handbook of numerical analysis, vol. 13, pp. 825-895, 2005.

[18] Avron, Haim, “A generalized Courant-Fischer minimax theorem,” 2014.

[19] Romijn, RC and Weiland, Siep and Marquardt, Wolfgang, "Proper orthogonal decomposition for model reduction of linear differentialalgebraic equation systems," in submitted to: 18th IFAC World Congress, 2011. 